\title{
Data Association - Solution or Avoidance : Evaluation of a Filter based on RFS framework and Factor Graphs with SME*
}

\author{
Dhiraj Gulati $^{1,2}$, Uzair Sharif ${ }^{2}$, Feihu Zhang ${ }^{3}$, Daniel Clarke ${ }^{4}$, and Alois Knoll ${ }^{2}$
}

\begin{abstract}
Data or measurement-to-track association is an integral and expensive part of any solution performing multitarget multi-sensor Cooperative Localization (CL) for better state estimation. Various performance evaluations have been performed between various state-of-the-art solutions, but they have been often limited within same family of algorithms. However, there exist solutions which avoid the task of data association to perform the $C L$ in a multi-target multi-sensor environment. Factor Graphs using Symmetric Measurement Equations (SMEs) factor is one such solution. In this paper we compare and contrast the state estimation using state-ofthe-art Random Finite Set (RFS) approach and using a Factor Graph solution with SMEs. For a RFS we use multi-sensor multi-object with the Generalized Labeled Multi-Bernoulli (GLMB) Filter. These two solution use conceptually different approaches, GLMB Filter solves the data association implicitly, but Factor Graph based solution avoids the task altogether. Simulations present an interesting results where for simple scenarios implemented GLMB filter performs efficiently. But the performance of GLMB Filter degrades faster than Factor Graphs using SMEs when the error in the sensors increase.
\end{abstract}

\section{INTRODUCTION}

With tumbling hardware costs, number of sensors available inside and outside the vehicle have increased. And along with the possibility of sharing data in real time has revolutionized the Vehicle to Vehicle (V2V) and Vehicle to Infrastructure (V2I) technologies. Hence, the task of Cooperative Localization to arrive at better state estimates of the participating vehicles has become a feasible solution [1].

Cooperative Localization (CL) is an old concept. [2] and [3] use Kalman Filter and its derivatives to perform the CL. Other researches provided novel solutions including Maximum A Posteriori Estimation (MAP) [4], Particle Filters [5], Markov localization [6], Split Covariance Intersection Filter [7], and Random Finite Set framework (RFS) [8].

Howard et. el. [9] uses Maximum Likelihood Estimation (MLE) to achieve the CL by combining relative measurements between robots in a least square formulation and

\footnotetext{
*This work was supported by fortiss http://www.fortiss.org/, BMWi IKT III SADA Project http://www.projekt-sada.de/ and BMVI Providentia http://testfeld-a9.de/.

${ }^{1}$ Dhiraj Gulati is with the fortiss $\mathrm{GmbH}$, München, gulatiefortiss.org

${ }^{2}$ Dhiraj Gulati, Uzair Sharif and Alois Knoll are with Technische Universität München, Garching bei München, Germany, dhiraj.gulati@tum.de, uzair.sharifetum.de, knollein.tum.de

${ }^{3}$ Feihu Zhang is with the School of Marine Science and Technology, Northwestern Polytechnical University, 710072, Xi'an, China; feihu.zhang@nwpu.edu.cn

${ }^{4}$ Daniel Clarke is with Cogsense Technologies Limited, Berkshire, RG14 1QL England, UK; daniel.clarke@cogsense.co.uk
}

solving the same. Ahmad et. el. [10] does the same but also includes moving landmarks. Gulati et. el. [11] formulates the $\mathrm{CL}$ as a graphical model, adding sensor measurements as SMEs to formulate the joint state of the system. This is implemented as a factor graph within the Georgia Tech Smoothing and Mapping (GTSAM) [12] framework.

Most of the CL methodologies also use some of the popular state-of-the-art solution to manage the data origin uncertainty. These techniques mostly fall in the following categories: Nearest Neighbor (NN) methods, Probabilistic Data Association (PDA) methods (like Joint PDA [13], [14] and Joint Integrated PDA [15]), Multiple Hypothesis Tracking (MHT) [16]-[18], multi-target particle filtering [19] and lately methods based on RFSs [20]-[23].

Some researchers have come up with other interesting methodologies to address the issue of data association. Gidel et. al. [24] demonstrated the used non-parametric data association for particle filter. Instead of the Gaussian Framework, particle set is generally non-Gaussian and authors propose non-parametric approaches of Parzen Window and K-Nearest Neighbour to compute probabilities of each data association. Bowman et. al. [25] formulate the problem of data association as part of the Factor Graph. The landmarks recorded in the system are identified using geometric and semantic information. The process assign weights to the subsequent measurements of the landmarks. The resulted graph is optimised using GTSAM [12].

In this evaluation, we use RFS based algorithm applied to a multi sensor multi target scenario. In the last decade researchers have increasingly developed a lot of novel approaches using family of RFS algorithms. For our comparisons we use a fast implementation of Generalized Labeled Multi-Bernoulli (GLMB) Filter [26].

Our previous work [11] using Factor Graph and SME successfully solves the problem of CL. It avoids data association and also scales optimally for non-linear cases.

\section{PROBlem DESCRIPTION}

The scenario which we use to compare the two methods shown in (Fig. 1(a)). The assumptions are:

1) Each vehicle is equipped with an Odometry and a GPS sensor and can broadcasts its measurements. Further GPS Sensor can localize itself in an absolute reference.

2) An external infrastructure RADAR sensor can derive the global position of all the vehicles in its field of view, but cannot uniquely identify the vehicles. This introduces a challenge from the perspective of data association, which is the main focus of our comparison. 


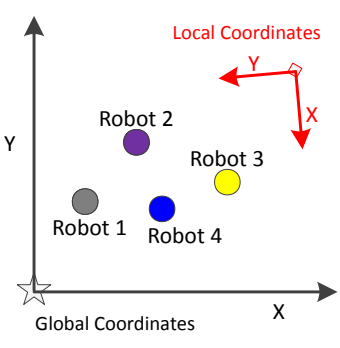

(a)

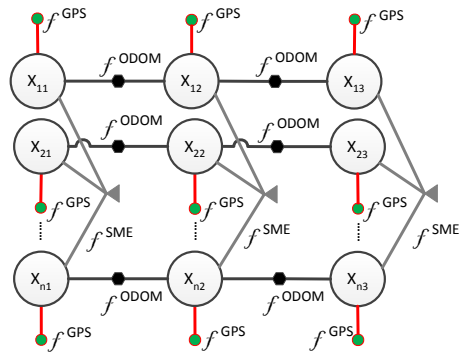

(b)
Fig. 1. (a) Four vehicles represented with solid circles. Local Coordinate System in red is of RADAR. (b) Factor graph for $n$ vehicles with three state nodes each and corresponding Odometry Factors, GPS Factors and SME Factors.

3) The vehicles and RADAR Sensor can communicate in both directions without any timing delay or data error.

4) The system has no clutter and no miss detections.

We use cooperative multi-sensor (Odometry, GPS, RADAR) and multi-target (for RADAR) data fusion to arrive at the state estimates of the participating vehicles.

\section{FACTOR GRAPHS AND SMES}

This sections gives a brief overview of Factor Graphs and SMEs. Interested reader should refer to [11] for details.

\section{A. Factor Graphs}

Localization can be represented by estimating the trajectory $\mathbf{x}=\left\{x_{i} \mid i \in 0, \ldots, n\right\}$, for a given set of measurements from various sensors, for example from Sensor-1 $\mathbf{z}^{1}=$ $\left\{\mathbf{z}^{1} \mid i \in 0, \ldots, n\right\}$, Sensor-2 $\mathbf{z}^{2}=\left\{z^{2} \mid i \in 0, \ldots, n\right\}$ and Odometry $\mathbf{u}=\{u \mid i \in 0, \ldots, n\}$. The joint density for the measurements from all the Sensors can be then written as:

$$
P\left(\mathbf{x}, \mathbf{z}^{1}, \mathbf{z}^{2}, \mathbf{u}\right) \propto P\left(x_{0}\right) \prod_{i}^{n} P\left(x_{i+1} \mid x_{i}, u_{i}\right) \prod_{k}^{m} P\left(z_{k} \mid x_{i k}\right)
$$

where $z_{k} \in\left\{\mathbf{z}^{1}, \mathbf{z}^{2}\right\}$ denotes the measurement, originating from either Sensor-1 or Sensor-2. This can also be represented as a Factor Graph [27] where factors are formulated from sensors measurements and connect the states.

To arrive at the optimized states, we calculate the maximum likelihood estimation (MLE) by using the nonlinear least square method:

$$
\begin{aligned}
& \overline{\mathbf{x}}=\underset{\operatorname{argmax}}{\arg } P\left(x_{i} \mid \mathbf{z}, \mathbf{u}\right)= \\
& \operatorname{argmin}\left\{\sum_{i=1}^{n}\left\|f_{i}\left(x_{i-1}-u_{i}\right)-x_{i}\right\|_{\Gamma_{i}}^{2}+\sum_{k=1}^{m}\left\|h_{k}\left(x_{i k}\right)-z_{k}\right\|_{\Sigma_{k}}^{2}\right\}
\end{aligned}
$$

where $h$ and $f$ denote the measurement and process models, and $\Gamma_{i}$ and $\Sigma_{k}$ are the covariance matrices. Next we look at the formulations of factors.

For a constant velocity model, the error function of the binary factor $f^{O D O M}$ between the states $X_{t}, X_{t-1}$ :

$$
f^{O D O M}\left(X_{t}, X_{t-1}\right) \triangleq d\left(z_{t}^{o}-h^{o}\left(z_{t-1}^{o}\right)\right)
$$

where $h^{o}$ is the function to calculate the odometry measurement at time $t$ and $z_{t}^{o}$ is the measurement at time $t$. The covariances are provided by the sensor manufacturer.
The GPS gives an unary factor $f^{G P S}$ for a state $X_{t}$, which is written as:

$$
f^{G P S}\left(X_{t}\right) \triangleq d\left(z_{t}^{g}-h^{g}\left(z_{t}\right)\right)
$$

where $n^{g}$ is the measurement noise, and $h^{g}$ is the measurement function, providing the relation between the measurement $z_{t}^{g}$ and the position of the vehicle $z_{t}$ at time $t$. The covariances are provided by the sensor manufacturer.

To avoid Data Association, the RADAR measurements are incorporated using a $\mathrm{N}$-ary $\mathrm{SME}$ factor for states $\left(X_{0}, \cdots, X_{N}\right)_{t}$ at time $t$ as:

$$
f^{S M E}\left(\left(X_{0}, \cdots, X_{N}\right)_{t}\right) \triangleq d\left(z_{t}^{s}-h^{s}\left(z_{0}, \cdots, z_{N}\right)\right)
$$

where $h^{s}$ is the new measurement function and $z_{i}$ is the $i^{\text {th }}$ measurement. Fig. 1(b) shows the factor graph with three kinds of factors.

The SME measurement is a pseudo measurement, hence we also need to calculate the covariance for it. If $\sigma_{x}^{2}$ and $\sigma_{y}^{2}$ are the $X$ and $Y$ variances respectively for the RADAR, then we can write [28]:

$$
\sigma_{t o p_{x, y}}^{2}=M \cdot \operatorname{Cov}(x, y) \cdot M^{T}
$$

where $M$ is a $1 X 2 N$ matrix as follows:

$$
M=\left[\frac{\partial}{\partial x_{1}}\left(z_{t}^{s}\right), \cdots, \frac{\partial}{\partial x_{n}}\left(z_{t}^{s}\right), \frac{\partial}{\partial y_{1}}\left(z_{t}^{s}\right), \cdots, \frac{\partial}{\partial y_{n}}\left(z_{t}^{s}\right)\right]
$$

\section{B. Smoothing}

The formulated factor graph is solved using the Levenberg Marquardt Optimizer. Using an initial estimate $x_{0}$ it iteratively finds an update $\Delta$ from the linearized system:

$$
\underset{\Delta}{\arg \min } J\left(x_{0}\right) \Delta-b\left(x_{0}\right)
$$

where $J\left(x_{0}\right)$ is the sparse Jacobian Matrix at the current linearization point $x_{0}$ and $b\left(x_{0}\right)=f\left(x_{0}\right)-z$ is the residual for given the measurement $z$. Further detail on this process is presented within [11] and [29].

\section{RANDOM Finite SETS (RFS) AND GLMB FILTER}

This section gives a brief overview of RFS and GLMB Filter. Interested reader should refer to [26], [30] for details.

\section{A. RFS}

Mathematically, the RFS framework lumps together all the objects' states and observations into finite-sets referred to as multi-object state $\boldsymbol{X}$ and multi-object observation $\boldsymbol{Z}$ at any time $k$. These sets are random in the number of elements, while each element of such a set is modelled via a randomvariable in the single-object state-space $\mathbb{X}$. This unifying notion of entire system's state and observation enables the application of Bayesian-Inferential Statistics in the derivation of optimal multi-object Bayes prediction-update recursion. Denoting the multi-state prediction and filtering densities by $\bar{\pi}_{+}(\boldsymbol{X}), \pi_{+}(\boldsymbol{X})$ respectively, the recursion is given by:

$$
\begin{gathered}
\overline{\boldsymbol{\pi}}_{+}(\boldsymbol{X})=\int f(\boldsymbol{X} \mid .) \boldsymbol{\pi}(.) \mu_{s}(d \boldsymbol{X}) \\
\boldsymbol{\pi}_{+}(\boldsymbol{X})=\frac{g(\boldsymbol{Z} \mid \boldsymbol{X}) \overline{\boldsymbol{\pi}}_{+}(\boldsymbol{X})}{\int g(\boldsymbol{Z} \mid .) \overline{\boldsymbol{\pi}}_{+}(.) \mu_{s}(d \boldsymbol{X})}
\end{gathered}
$$


where $f\left(\boldsymbol{X}_{+} \mid \boldsymbol{X}\right)$ is the multi-object transition-density, and $g(\boldsymbol{Z} \mid \boldsymbol{X})$ is the multi-object likelihood function extracted from the assumed system dynamics-model and sensor-model respectively. The $\mu_{s}$ is an appropriate reference measure on the RFS state-space denoted by $\mathcal{F}(\mathbb{X})$. Because of the multiple integrals on $\mathcal{F}(\mathbb{X})$, the optimal Bayes filter is only computationally tractable for very small number of objects within the system.

\section{B. GLMB Filter}

Under the assumptions of standard multi-object transition and observation models, Vo et al. in [31] have shown that a special case of labelled RFS called the GLMB RFS enables an exact solution to (9), (10). Mathematically a GLMB RFS is characterized by the density:

$$
\boldsymbol{\pi}(\boldsymbol{X})=\Delta(\boldsymbol{X}) \sum_{(I, \xi) \in \mathcal{F}(\mathbb{L}) \times \Xi} w^{(I, \xi)} \delta_{I}(\mathcal{L}(\boldsymbol{X}))\left[p^{(\xi)}\right]^{\boldsymbol{X}}
$$

where $\Xi$ is a discrete space. Essentially (11) gives GLMB density as a mixture of multi-object exponentials where each component is identified by a hypothesis $(I, \xi)$ with weight $w^{(I, \xi)}$. As shown in [32], given a GLMB initial density all subsequent multi-object densities are GLMBs and can be computed exactly by a tractable recursion. This recursion starts with a prediction step given by:

$$
\begin{aligned}
\overline{\boldsymbol{\pi}}_{+}(\mathbf{X})=\Delta & (\mathbf{X}) \sum_{\xi, J, L_{+}} \bar{\omega}_{+}^{\left(\xi, J, L_{+}\right)} \delta_{J \cup L_{+}}[\mathcal{L}(\mathbf{X})]\left[\bar{p}_{+}^{(\xi)}\right]^{\mathbf{X}} \\
\bar{\omega}_{+}^{\left(\xi, J, L_{+}\right)} & =1_{\mathcal{F}\left(\mathbb{B}_{+}\right)}\left(L_{+}\right) r_{B,+}^{L_{+}}\left[1-r_{B,+}\right]^{\mathbb{B}_{+}-L_{+}} \\
& \times \sum_{I \in \mathcal{F}(\mathbb{L})} 1_{\mathcal{F}(I)}(J)\left[\bar{P}_{S}^{(\xi)}\right]^{J}\left[1-\bar{P}_{S}^{(\xi)}\right]^{I-J} \omega^{(I, \xi)} \\
\bar{P}_{S}^{(\xi)}(\ell) & =\left\langle p^{(\xi)}(\cdot, \ell), P_{S}(\cdot, \ell)\right\rangle \\
\bar{p}_{+}^{(\xi)}\left(x_{+}, \ell_{+}\right) & =1_{\mathbb{L}}\left(\ell_{+}\right) \frac{\left\langle P_{S}\left(\cdot, \ell_{+}\right) f_{+}\left(x_{+} \mid \cdot, \ell_{+}\right), p^{(\xi)}\left(\cdot, \ell_{+}\right)\right\rangle}{\bar{P}_{S}^{(\xi)}\left(\ell_{+}\right)} \\
& +1_{\mathbb{B}_{+}}\left(\ell_{+}\right) p_{B,+}\left(x_{+}, \ell_{+}\right) .
\end{aligned}
$$

The recursion continues by applying the measurementcorrection on the predicted density to compute the filtering density as:

$$
\begin{aligned}
& \boldsymbol{\pi}_{+}(\mathbf{X}) \propto \Delta(\mathbf{X}) \sum_{\xi, J, L_{+}, \theta_{+}} \omega_{Z_{+}}^{\left(\xi, J, L_{+}, \theta_{+}\right)} \delta_{J \cup L_{+}}[\mathcal{L}(\mathbf{X})] \\
& \times\left[p_{Z_{+}}^{\left(\xi, \theta_{+}\right)}\right]^{\mathbf{X}} \\
& \omega_{Z_{+}}^{\left(\xi, J, L_{+}, \theta_{+}\right)}=1_{\Theta_{+}\left(J \cup L_{+}\right)}\left(\theta_{+}\right)\left[\bar{\psi}_{Z_{+}}^{\left(\xi, \theta_{+}\right)}\right]^{J \cup L_{+}} \bar{\omega}_{+}^{\left(\xi, J, L_{+}\right)} \\
& \bar{\psi}_{Z_{+}}^{\left(\xi, \theta_{+}\right)}\left(\ell_{+}\right)=\left\langle\bar{p}_{+}^{(\xi)}\left(\cdot, \ell_{+}\right), \psi_{Z_{+}}^{\left(\theta_{+}\left(\ell_{+}\right)\right)}\left(\cdot, \ell_{+}\right)\right\rangle \\
& p_{Z_{+}}^{\left(\xi, \theta_{+}\right)}\left(x_{+}, \ell_{+}\right)=\frac{\bar{p}_{+}^{(\xi)}\left(x_{+}, \ell_{+}\right) \psi_{Z_{+}}^{\left(\theta_{+}\left(\ell_{+}\right)\right)}\left(x_{+}, \ell_{+}\right)}{\bar{\psi}_{Z_{+}}^{\left(\xi, \theta_{+}\right)}\left(\ell_{+}\right)}
\end{aligned}
$$

To compute this recursion we make use of the GaussianMixture implementation of the GLMB filter as provided in [21]. This implementation assumes linear-Gaussian constraints on target dynamics and sensor observations. Under these assumptions, the individual target states take on the form of Gaussian Mixtures on $\mathbb{X}$.

\section{Evaluation}

\section{A. Simulation Setup}

To compare the two approaches, we set an simulation of two vehicles on a ground plane. The factor graphs and corresponding factors are implemented using GTSAM open source library [12]. We use GLMB Filter [26] to implement the RFS solution. The tests are coded in $\mathrm{C}++$ and run on an Ubuntu 16.04 LTS 64-bit machine with 16 GB RAM and Intel(R) Core(TM) i7-4710MQ CPU @ 2.50GHz processor.

Simulated vehicles are assumed to have Odometry and GPS Sensors. GPS Sensor provides the location in global coordinates. The infrastructure RADAR is mounted such that it is able to observe the vehicles for the complete trajectory. We assume its location is known and provides the measurement in global coordinate without performing any data association. The measurement noises are assumed to be Gaussian. The covariance are assumed as diag[1.0,1.0], $\operatorname{diag}[9.0,9.0]$ and $\operatorname{diag}[0.1,0.1]$ for the Odometry, the GPS and the RADAR respectively.

As outlined in the initial assumptions we don't have any miss detection or clutter, therefore the detection probability for GLMB $P_{d}=1.0$. Infrastructure sensor is able to observe the entire trajectory, the survival probability $P_{s}=1.0$. The multi-sensor GLMB filter is implemented via the Gibbs sampling technique, the filter is configured with maximum association hypothesis $H^{\max }=100$. Further the GLMB [26] is a Gaussian Mixture implementation, that assumes that the targets move with a constant velocity model.

Results from the simulation are compared three ways, between:

- the fused trajectory for GLMB using GPS and RADAR; and

- the fused trajectory for Odometry, GPS, SME Factor (constructed using RADAR) [11].

Current implementation of GLMB Filter assumes the measurements from the sensors as the target positions in the global coordinate framework. On the other hand, Odometry is only the difference between the two consecutive states of the vehicles. Using the cumulative Odometry to calculate the position increases the covariance for each subsequent step. This results in the failure of the tracker. Hence we did not use the Odometry for our final comparison for GLMB Filter. Further we also compare with two other covariances of the RADAR as $[3,3]$ and $[5,5]$.

The performance of the algorithms is measured by Optimal Sub-pattern Assignment (OSPA). This was proposed first in [33] and calculates the miss-distance and the corresponding error between the calculated and estimated individual target states. If $X$ is the set of estimated states with cardinality $m$ and $Y$ is the set of true target states with cardinality $n$, then 


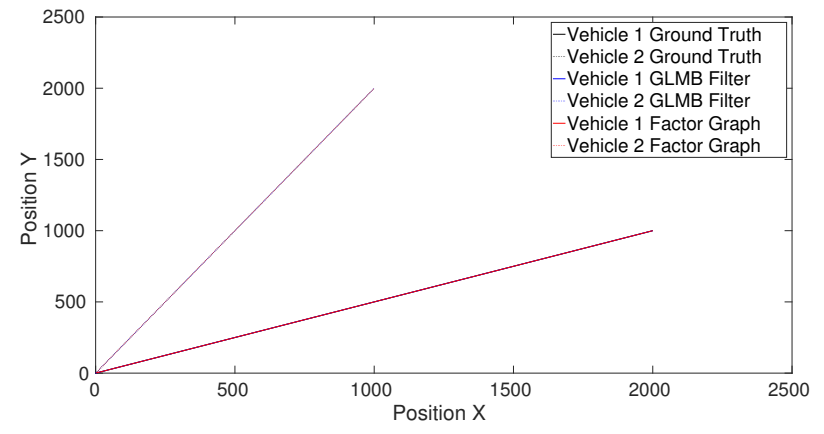

Fig. 2. Trajectories of the two vehicles showing Ground Truth, and calculated from GLMB Filter and Factor Graphs with RADAR covariance as $[.1, .1]$.

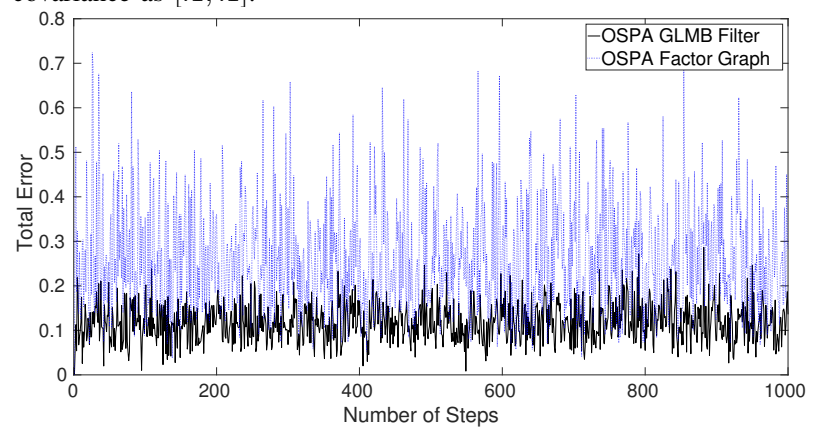

Fig. 3. OSPA for fused trajectories from GLMB Filter and Factor Graph for RADAR covariance $[.1, .1]$.

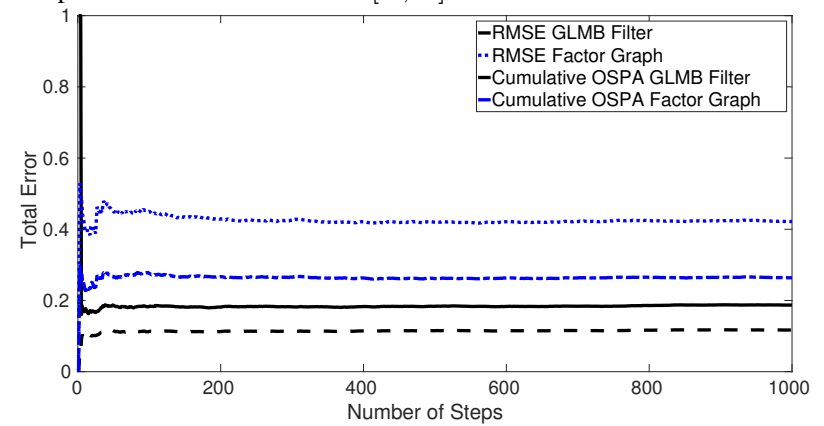

Fig. 4. Total system RMSE and Cumulative OSPA for fused trajectories from GLMB Filter and Factor Graph for RADAR covariance $[.1, .1]$.

OSPA measure is defined as:

$$
d_{p}^{c}(X, Y)=\left(\frac{1}{n}\left(\min \sum_{i=1}^{m} d^{c}\left(x_{i}, y_{\pi(i)}\right)^{p}+c^{p}(n-m)\right)\right)^{1 / p}
$$

if $m \leq n$ and $d_{p}^{c}(X, Y)=d_{p}^{c}(Y, X)$ otherwise. Here, $d^{c}(x, y) \triangleq \min (c, d(x, y))$ is the distance between $x$ and $y$, cut-off at $c$. For our comparison we have kept $c$ as 100.

The performance is also measured by calculating Root Mean Square Error (RMSE) value for the complete system. The total error is the sum of the RMSE of each vehicle for $n$ steps:

Error $=\sqrt{\frac{\sum_{j=1}^{n} \sum_{i=1}^{2}\left[\left(x_{i_{e s t}}-x_{i_{\text {real }}}\right)^{2}+\left(y_{i_{\text {est }}}-y_{i_{\text {real }}}\right)^{2}\right]^{j}}{n}}$

\section{B. Results}

Figure 2 shows the Ground Truth and fused trajectories using GLMB Filter and Factor Graphs. Since the fused

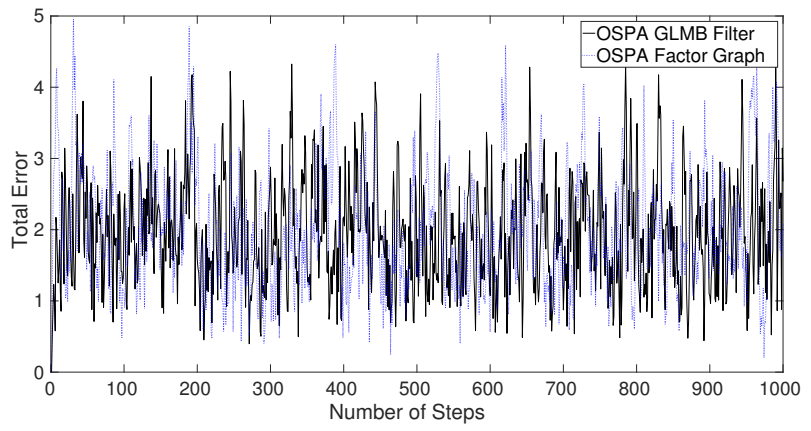

Fig. 5. OSPA for fused trajectories from GLMB Filter and Factor Graph for RADAR covariance [3,3].

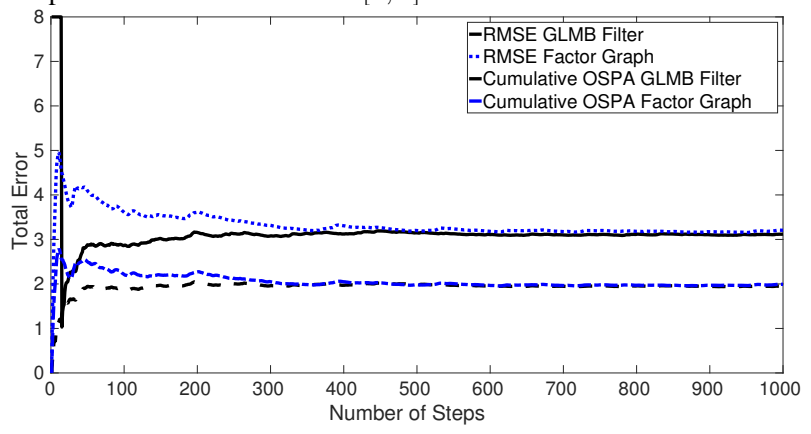

Fig. 6. Total system RMSE and Cumulative OSPA for fused trajectories from GLMB Filter and Factor Graph for RADAR covariance $[3,3]$.

trajectories are very near to the Ground Truth, it is difficult to analyse the performance. Therefore, Fig. 3 shows the OSPA for the 1000 steps. Again it becomes difficult to judge the performance. Hence we plot the RMSE for the same as shown in Fig. 4. It can be clearly seen GLMB Filter performs better than the Factor Graphs. For the first few steps, the RMSE is very high. This is intentionally kept high because GLMB Filter at beginning is unable to detect all the targets.

Now, GLMB Filter does not always detect the targets and also results in state estimates of the targets in a random order, hence calculating RMSE becomes challenging. Therefore we plot the cumulative OSPA which is calculated as:

$$
C-O S P A=\frac{\sum_{i=1}^{N} d_{p}^{c}}{N}
$$

where $d_{p}^{c}$ is defined in Eq. 20, and $N$ is the number of steps.

From Fig. 4, we can see that cumulative OSPA not only conveys the same information as RMSE but also does not suffer the problem of undetected targets and the correct order of the fused results. GLMB Filter performs quite optimally because it gives high weight to the RADAR measurements which have a very low covariance. On the other hand Factor Graph avoids the data association using SME Factor which is a derived pseudo-measurement. The corresponding covariance is also a derived one and is not able to match the native RADAR covariance.

To further evaluate the differences, we change the covariance of the RADAR to $[3,3]$. Figure 5 shows the OSPA and Fig. 6 shows the RMSE and cumulative OSPA for the same. It can be seen both the methods perform almost similarly. Figure 7 shows the OSPA and Fig. 8 shows the 


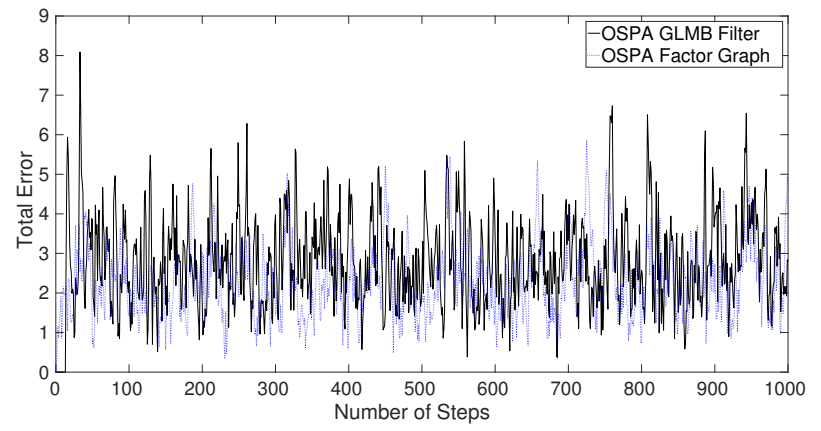

Fig. 7. OSPA for fused trajectories from GLMB Filter and Factor Graph for RADAR covariance $[5,5]$.

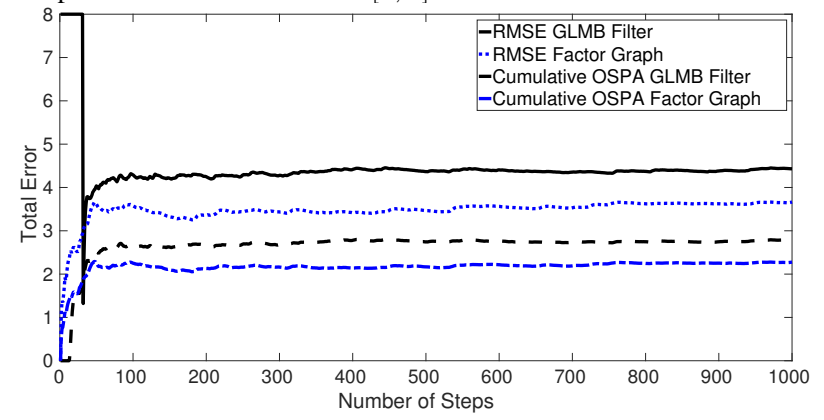

Fig. 8. Total system RMSE and Cumulative OSPA for fused trajectories from GLMB Filter and Factor Graph for RADAR covariance $[5,5]$.

RMSE and cumulative OSPA for the RADAR covariance of $[5,5]$. With increase in the RADAR covariance, the GLMB Filter degrades faster than Factor Graphs. This is because Factor Graph also uses the Odometery and hence this extra information keeps the degradation in check.

To further evaluate the stability of both the solutions we run a Monte Carlo Simulation of 1000 iterations for 2, 3 and 4 targets. Here we compare using the above proposed $\mathrm{Cu}$ mulative OSPA, as already mentioned above that calculating RMSE for GLMB Filter is a challenging task. Table I shows the corresponding results rounding upto three decimal places.

TABLE I

Average Final Cumulative OSPA VALUes For 1000 Iterations

\begin{tabular}{ccccccc}
\hline \multirow{2}{*}{ Total targets } & \multicolumn{3}{c}{ GLMB Filter } & \multicolumn{3}{c}{ Factor Graph } \\
\cline { 2 - 7 } & {$[.1, .1]$} & {$[3,3]$} & {$[5,5]$} & {$[.1,1]$} & {$[3,3]$} & {$[5,5]$} \\
\hline & & & & & & \\
2 & .116 & 1.952 & 2.719 & .263 & 1.987 & 2.221 \\
3 & .116 & 1.958 & 2.728 & .888 & 1.996 & 2.232 \\
4 & .116 & 1.951 & 2.721 & .733 & 1.978 & 2.221 \\
\hline
\end{tabular}

As can be seen from the Table I, with an increase in the targets, the system performance remains stable. It reflects the results discussed for a system of 2 targets. Next we analyse the execution performance by running Monte Carlo Simulation of 1000 iterations for 2,3 and 4 targets. Table II shows the corresponding results in seconds rounding upto four decimal places.

As can be seen from the Table II, GLMB Filter takes less time for all the cases of 2,3 and 4 targets. This is expected because Factor Graph based solution currently
TABLE II

AVERAGE FINAL EXECUTION TIME IN SECONDS FOR 1000 ITERATIONS

\begin{tabular}{ccccccc}
\hline \multirow{2}{*}{ Total targets } & \multicolumn{3}{c}{ CLMB Filter } & \multicolumn{3}{c}{ Factor Graph } \\
\cline { 2 - 7 } & {$[.1, .1]$} & {$[3,3]$} & {$[5,5]$} & {$[.1, .1]$} & {$[3,3]$} & {$[5,5]$} \\
\hline & & & & & & \\
2 & .0602 & .0603 & .0602 & .1942 & .1249 & .1220 \\
3 & .1257 & .1260 & .1236 & .3546 & .2244 & .2109 \\
4 & .2252 & .2245 & .2229 & .5904 & .3290 & .3213 \\
\hline
\end{tabular}

uses offline batch optimization smoother which solves the complete graph. On the other hand, GLMB being a filter only calculates the current state, thereby executing faster. The table shows an anomaly as well. The Factor Graph for 3 vehicles seem to perform better than 2 or 4 . But we did not see any such behaviour in our past results in [11].

In our simulations, original measurements from the RADAR have been used, hence data association is avoided in Factor Graph with SME method or is solved internally by GLMB Filter. Instead of various states and covariances with each time step, only measurements are sent, keeping the bandwidth requirements to minimum for both the solutions.

\section{Remarks}

Although we have successfully avoided the data association issue in the RADAR, the current results assume clutter free environment. In practice this is not the case. GLMB Filter is capable to perform under the clutter scenario while Factor Graph solution would need additional solutions like [34], [35] to tackle the challenge.

Currently GLMB Filter is a Gaussian Mixture implementation, that assumes that the targets move with a constant velocity model. Hence it can only handle targets travelling in relative straight trajectories. For complex scenarios like constant turn or acceleration and random trajectories, additional solutions like Particle Filter have to be used. But the Factor Graph based solution does not require any additional solution to handle complex scenarios. But using particle filter may increase the average execution time.

For online real scenarios online incremental smoothing algorithm like iSAM2 [36] (supported in GTSAM) is required. Further the GTSAM framework supports the notion of plug and play [37] thereby providing a efficient platform for scenarios when all the measurements are not present from all the sensors. This is not possible for GLMB Filter.

\section{CONCLUSION}

In this paper we evaluate two state-of-the-art methods which are used to address the data association challenge for multi-target multi-sensor scenarios. On one hand we estimate the states using multi-sensor multi-object with the Generalized Labeled Multi-Bernoulli (GLMB) Filter and and on the other hand use a factor graph solution using SMEs for cooperative localization. The performance is primarily assessed by cumulative OSPA with upto 4 targets.

In this evaluation we find that GLMB Filter performs better when the error covariance of the RADAR sensor is low. But with an increase in the error covariance the 
performance for GLMB Filter degrades faster than the Factor Graph with SME. GLMB Filter has lower execution time when compared to Factor Graph solution. But this is expected as we use the offline batch optimization for Factor Graph.

Both the solutions address the challenges of bandwidth issue and scalability for the given test scenarios. But it warrants further testing with highway like scenarios where the number of vehicles can range between $20-40$.

Future research work will focus on the comparison when influence of clutter and miss-detections is present like in a real-world system. It will also consider comparing implementations incorporating other models like constant turn and constant acceleration and random trajectories for both the solutions. Additionally online incremental smoothing algorithm will be used for comparisons.

\section{REFERENCES}

[1] R. Kurazume, S. Nagata, and S. Hirose, "Cooperative positioning with multiple robots," in Robotics and Automation, 1994. Proceedings., 1994 IEEE International Conference on, May 1994, pp. 1250-1257 vol.2.

[2] S. I. Roumeliotis and G. A. Bekey, "Distributed multirobot localization," IEEE Transactions on Robotics and Automation, vol. 18, no. 5, pp. 781-795, Oct 2002.

[3] N. Karam, F. Chausse, R. Aufrere, and R. Chapuis, "Cooperative multi-vehicle localization," in 2006 IEEE Intelligent Vehicles Symposium. IEEE, 2006, pp. 564-570.

[4] E. D. Nerurkar, S. I. Roumeliotis, and A. Martinelli, "Distributed maximum a posteriori estimation for multi-robot cooperative localization," in Robotics and Automation, 2009. ICRA '09. IEEE International Conference on, May 2009, pp. 1402-1409.

[5] I. M. Rekleitis, G. Dudek, and E. E. Milios, "Multi-robot cooperative localization: a study of trade-offs between efficiency and accuracy," in IEEE/RSJ International Conference on Intelligent Robots and Systems, vol. 3, 2002, pp. 2690-2695 vol.3.

[6] D. Fox, W. Burgard, H. Kruppa, and S. Thrun, "A probabilistic approach to collaborative multi-robot localization," Autonomous robots, vol. 8, no. 3, pp. 325-344, 2000.

[7] H. Li and F. Nashashibi, "Cooperative multi-vehicle localization using split covariance intersection filter," IEEE Intelligent Transportation Systems Magazine, vol. 5, no. 2, pp. 33-44, Summer 2013.

[8] F. Zhang, H. Stähle, G. Chen, C. Buckl, and A. Knoll, "Multiple vehicle cooperative localization under random finite set framework," in 2013 IEEE/RSJ International Conference on Intelligent Robots and Systems, Nov 2013, pp. 1405-1411.

[9] A. Howard, M. J. Matark, and G. S. Sukhatme, "Localization for mobile robot teams using maximum likelihood estimation," in Intelligent Robots and Systems, 2002. IEEE/RSJ International Conference on, vol. 1, 2002, pp. 434-439 vol.1.

[10] A. Ahmad, G. D. Tipaldi, P. Lima, and W. Burgard, "Cooperative robot localization and target tracking based on least squares minimization," in 2013 IEEE International Conference on Robotics and Automation, May 2013, pp. 5696-5701.

[11] D. Gulati, F. Zhang, D. Clarke, and A. Knoll, "Graph-based cooperative localization using symmetric measurement equations," Sensors, vol. 17(6), no. 1422, 2017. [Online]. Available: http://www.mdpi.com/1424-8220/17/6/1422

[12] "GTSAM, Georgia Tech smoothing and Mapping," https://collab.cc.gatech.edu/borg/gtsam/.

[13] T. E. Fortmann, Y. Bar-Shalom, and M. Scheffe, "Multi-target tracking using joint probabilistic data association," in Decision and Control including the Symposium on Adaptive Processes, 1980 19th IEEE Conference on, Dec 1980, pp. 807-812.

[14] S. H. Rezatofighi, A. Milan, Z. Zhang, Q. Shi, A. Dick, and I. Reid, "Joint probabilistic data association revisited," in 2015 IEEE International Conference on Computer Vision (ICCV), Dec 2015, pp. 30473055.

[15] D. Musicki, R. Evans, and S. Stankovic, "Integrated probabilistic data association," IEEE Transactions on Automatic Control, vol. 39, no. 6, pp. 1237-1241, Jun 1994.
[16] D. Reid, "An algorithm for tracking multiple targets," IEEE Transactions on Automatic Control, vol. 24, no. 6, pp. 843-854, Dec 1979.

[17] R. L. Streit and T. E. Luginbuhl, "A probabilistic multi-hypothesis tracking algorithm without enumeration and pruning," in Proceedings of the Sixth Joint Service Data Fusion Symposium, 1993, pp. 10151024.

[18] S. S. Blackman, "Multiple hypothesis tracking for multiple target tracking," IEEE Aerospace and Electronic Systems Magazine, vol. 19, no. 1, pp. 5-18, Jan 2004.

[19] J. Vermaak, S. J. Godsill, and P. Perez, "Monte carlo filtering for multi target tracking and data association," IEEE Transactions on Aerospace and Electronic Systems, vol. 41, no. 1, pp. 309-332, Jan 2005.

[20] R. P. S. Mahler, "Multitarget bayes filtering via first-order multitarget moments," IEEE Transactions on Aerospace and Electronic Systems, vol. 39, no. 4, pp. 1152-1178, Oct 2003.

[21] B. N. Vo, B. T. Vo, and D. Phung, "Labeled random finite sets and the bayes multi-target tracking filter," IEEE Transactions on Signal Processing, vol. 62, no. 24, pp. 6554-6567, Dec 2014.

[22] R. Mahler, "A theory of phd filters of higher order in target number," in Defense and Security Symposium. International Society for Optics and Photonics, 2006, pp. 62 350K-62 350K.

[23] B. T. Vo, B. N. Vo, and A. Cantoni, "The cardinality balanced multitarget multi-bernoulli filter and its implementations," IEEE Transactions on Signal Processing, vol. 57, no. 2, pp. 409-423, Feb 2009.

[24] S. Gidel, C. Blanc, T. Chateau, P. Checchin, and L. Trassoudaine, "Nonparametric data association for particle filter based multi-object tracking: application to multi-pedestrian tracking," in 2008 IEEE Intelligent Vehicles Symposium, June 2008, pp. 73-78.

[25] S. L. Bowman, N. Atanasov, K. Daniilidis, and G. J. Pappas, "Probabilistic data association for semantic slam," in 2017 IEEE International Conference on Robotics and Automation (ICRA), May 2017, pp. 17221729.

[26] B. Ngu Vo and B. Tuong Vo, "Multi-Sensor Multi-object Tracking with the Generalized Labeled Multi-Bernoulli Filter," ArXiv e-prints, Feb. 2017.

[27] V. Indelman, S. Williams, M. Kaess, and F. Dellaert, "Information fusion in navigation systems via factor graph based incremental smoothing," Robotics and Autonomous Systems, vol. 61, no. 8, pp. 721-738, 2013.

[28] K. O. Arras, "An introduction to error propagation: Derivation, meaning and examples of equation cy $=$ fx cxfx t." ETH-Zürich, Tech. Rep., 1998.

[29] F. Dellaert and M. Kaess, "Square root sam: Simultaneous localization and mapping via square root information smoothing," Int. J. Rob. Res., vol. 25, no. 12, pp. 1181-1203, Dec 2006. [Online]. Available: http://dx.doi.org/10.1177/0278364906072768

[30] S. Reuter, B. T. Vo, B. N. Vo, and K. Dietmayer, "The labeled multibernoulli filter," IEEE Transactions on Signal Processing, vol. 62, no. 12, pp. 3246-3260, June 2014

[31] B. T. Vo and B. N. Vo, "Labeled random finite sets and multi-object conjugate priors," IEEE Transactions on Signal Processing, vol. 61, no. 13, pp. 3460-3475, July 2013.

[32] B. N. Vo, B. T. Vo, and H. G. Hoang, "An efficient implementation of the generalized labeled multi-bernoulli filter," IEEE Transactions on Signal Processing, vol. 65, no. 8, pp. 1975-1987, April 2017.

[33] D. Schuhmacher, B. T. Vo, and B. N. Vo, "On performance evaluation of multi-object filters," in 2008 11th International Conference on Information Fusion, June 2008, pp. 1-8.

[34] M. Baum and U. D. Hanebeck, "The kernel-sme filter for multiple target tracking," in Proceedings of the 16th International Conference on Information Fusion, July 2013, pp. 288-295.

[35] Y. J. Lee and E. W. Kamen, "Sme filter approach to multiple target tracking with false and missing measurements," pp. 1954 - 1954 13, 1993. [Online]. Available: http://dx.doi.org/10.1117/12.157788

[36] M. Kaess, H. Johannsson, R. Roberts, V. Ila, J. Leonard, and F. Dellaert, "isam2: Incremental smoothing and mapping with fluid relinearization and incremental variable reordering," in 2011 IEEE International Conference on Robotics and Automation, May 2011, pp. 3281-3288.

[37] H. P. Chiu, X. S. Zhou, L. Carlone, F. Dellaert, S. Samarasekera, and R. Kumar, "Constrained optimal selection for multi-sensor robot navigation using plug-and-play factor graphs," in 2014 IEEE International Conference on Robotics and Automation (ICRA), May 2014, pp. 663670 . 